\title{
ARTIGO ORIGINAL \\ Construção participativa de uma gerontotecnologia sobre acidente vascular cerebral
}

Geisieli Maria Sgrignoli Dias*, Célia Maria Gomes Labegalini**, Marina Bennemann de Moura, M.Sc. ${ }^{* *}$, Isabela Vanessa Tavares Cordeiro Silva ${ }^{* \star *}$, Lígia Carreira, D.Sc. ${ }^{* \star * *}$, Vanessa Denardi Antoniassi Baldissera, D.Sc..****

${ }^{*}$ Enfermeira pela Universidade Estadual de Maringá, Maringá/PR, ${ }^{* * D o u t o r a n d a ~ e m ~}$ Enfermagem pela Universidade Estadual de Maringá, ${ }^{* \star *}$ Enfermeira da Unidade de Pronto Atendimento Zona Sul de Maringá/PR, ${ }^{* * * *}$ Graduanda em Enfermagem pela Universidade Estadual de Maringá/PR, ${ }^{* * * * * D o c e n t e ~ d o ~ D e p a r t a m e n t o ~ d e ~ E n f e r m a g e m ~ d a ~ U n i v e r s i d a d e ~}$ Estadual de Maringá/PR

Recebido em 24 de agosto de 2017; aceito em 1 de junho de 2018.

Endereço de correspondência: Célia Maria Gomes Labegalini, Universidade Estadual de Maringá PR, Av. Colombo, 5790 Bloco 2/Sala 1, 87020-110 Maringá PR, E-mail: celialabegalini-@hotmail.com; Geisieli Maria Sgrignoli Dias: geize_dias@hotmail.com; Marina Bennemann de Moura: marinabenn@hotmail.com; Isabela Vanessa Tavares Cordeiro Silva: iza_bela_bela@hotmail.com; Lígia Carreira: ligiacarreira.uem@gmail.com; Vanessa Denardi Antoniassi Baldissera: vanessadenardi@hotmail.com

\section{Resumo}

Introdução: O envelhecimento populacional é um fato marcante neste século e impactou na organização social e dos serviços de saúde, necessitando de ampliação dos serviços prestados a este grupo etário especialmente no que se refere à educação e promoção da saúde. Objetivo: Descrever o processo contextualizado e participativo da construção de uma gerontotecnologia educativa na atenção básica sobre acidente vascular cerebral. Material e métodos: Pesquisa de desenvolvimento metodológico, com abordagem qualitativa, elaborada a partir das demandas de idosos usuários de uma unidade básica de saúde. Foi construído um vídeo educativo validado por experts na área e por idosos. Resultados: Os resultados foram organizados em: a elaboração da gerontotecnologia educativa; a adequação por juízes; e roda de conversa e adequação pelos idosos participantes. A elaboração participativa e dialogada de uma gerontotecnologia auxilia no processo de ensino-aprendizagem dado seu potencial educativo, por pautar-se nas necessidades reais dos idosos. Conclusão: O percurso metodológico de construção e adequação da gerontotecnologia educativa na temática acidente vascular cerebral mostrou-se oportuna e possível para abordagem de idosos em sala de espera de unidades básicas de saúde.

Palavras-chave: idosos, participação do paciente, Enfermagem, tecnologia educacional.

\begin{abstract}
Participatory construction of a gerontechnology on stroke

Introduction: Population aging is a marked fact in this century and has influenced the social organization and health services, therefore, an increase in the services provided to this age group especially in what concerns education and health promotion is needed. Objective: To describe the contextualized and participatory process of the construction of an educational gerontechnology in the basic attention on stroke. Methods: Methodological research with a qualitative approach, which was elaborated from the demands of elderly users of a Basic Health Unit. An educational video was constructed and validated by experts in the field and by the elderly. Results: The results are organized in: the elaboration of educational gerontechnology, the adequacy by judges, and round of conversation and adaptation by the elderly participants. The participatory and dialogued elaboration of a gerontechnology helps the teaching-learning process given its educational potential, because was based on the real needs of the elderly. Conclusion: The methodological course of construction and adequacy of the educational gerontechnology in the subject of cerebral vascular accident proved to be opportune and possible to approach the elderly in the waiting room of basic health units.
\end{abstract}

Key-words: aged, patient participation nursing, educational technology. 


\section{Resumen}

Construcción participativa de una gerontotecnología sobre accidente vascular cerebral

Introducción: El envejecimiento de la población es un hecho notable en este siglo e impactó en la organización social y de los servicios de salud, necesitando de ampliación de los servicios prestados a este grupo de edad especialmente en lo que se refiere a la educación y promoción de la salud. Objetivo: Describir el proceso contextualizado y participativo de la construcción de una gerontotecnología educativa en la atención básica sobre accidente vascular cerebral. Material y métodos: Investigación de desarrollo metodológico, con abordaje cualitativo. Elaborada a partir de las demandas de personas mayores usuarias de una Unidad Básica de Salud. Se construyó un video educativo validado por expertos en el área y por ancianos. Resultados: Los resultados fueron organizados en: la elaboración de la gerontotecnología educativa; la adecuación por jueces; y rueda de conversación y adecuación por los adultos mayores participantes. La elaboración participativa y dialogada de una gerontotecnología auxilia en el proceso de enseñanza-aprendizaje dado su potencial educativo, por pautarse en las necesidades reales de los adultos mayores. Conclusión: El recorrido metodológico de construcción y adecuación de la gerontotecnología educativa en la temática accidente vascular cerebral se mostró oportuna y posible para el abordaje de mayores en sala de espera de Unidades Básicas de Salud.

Palabras-clave: anciano, participación del paciente, Enfermería, tecnología educacional.

Introdução

O envelhecimento populacional é um fato marcante do século XXI em todo o mundo. No Brasil, a população idosa - indivíduos com mais de 60 anos - são o segmento populacional que mais aumenta, com taxas de crescimento de mais de $4 \%$ ao ano, o que totalizará no ano de 2030, cerca de 41,5 milhões de pessoas e 73,5 milhões em $2060[1,2]$.

Essa alteração demográfica se deu pela evolução científica, tecnológica e social que ocorreu no século passado e levou ao aumento na expectativa de vida e diminuição da morbimortalidade, que aliados à diminuição da natalidade levam ao progressivo envelhecimento da população mundial. Esse novo contingente populacional alterou as necessidades sociais e de saúde da população, tornando-se um grande desafio organizar os serviços de saúde para atender a população idosa, mantê-la saudável, ativa e promover sua saúde $[2,3]$.

A longevidade é um fenômeno que reflete a qualidade da assistência à saúde, contudo o país possui alta incidência e mortalidade por doenças evitáveis e crônicas, como as cardiovasculares, as quais são responsáveis por $29,4 \%$ das mortes do país, sendo os homens com idade média de 56 anos os principais acometidos. Estas doenças acometem o coração e as artérias e as principais são: infarto agudo do miocárdio e acidente vascular cerebral, além de arritmias cardíacas, isquemias ou anginas [4,5]. Desta forma, as doenças cardiovasculares causam grande impacto nos indicadores de saúde e na qualidade de vida da população, necessitando de ações de saúde específicas e eficientes.

Dentre as ações de cuidado ao idoso e as condições crônicas, a educação emerge no contexto da saúde como estratégia adequada para a sua promoção, desde que voltada às necessidades dos indivíduos e com abordagem adequada ao público. As atividades educativas permitem a elaboração e o fortalecimento da cumplicidade e do vínculo entre equipe de saúde e população e devem priorizar a autonomia e o empoderamento dos indivíduos. Desta forma, as práticas educativas integram-se aos cuidados de saúde e de enfermagem para construir coletivamente os saberes relativos ao processo saúde-doença-cuidado [3,6].

A educação no âmbito da saúde é considerada como uma estratégia de transformação de práticas e melhoria do atendimento e da qualidade de vida. O cotidiano da enfermagem é permeado por diversas práticas educativas, que vão desde a comunicação e empoderamento dos idosos no âmbito dos serviços de saúde à criação e desenvolvimento de softwares e materiais educativos, e estes são nomeados de tecnologias educionais [6,7]. Quando se refere ao público idoso, existe uma nomenclatura nova e própria para as tecnologias educativas, intitulada de gerontotecnologia, desenvolvida com a união dos conceitos de tecnologia, gerontologia e complexidade [7].

Assim, gerontotecnologia é termo mundialmente utilizado e conceitua todo produto, processo, estratégia, serviço e/ou conhecimento, desenvolvido com objetivo de promover a autonomia de idosos tendo como público-alvo os próprios idosos e/ou seus familiares e cuidadores. No âmbito da educação e da saúde é denominada de gerontotecnologia cuidativo- 
educacional, por seu potencial de contribuir e auxiliar no cuidado e na saúde do idoso. Além do público-alvo específico, as gerontotecnologias, por seu caráter de promover autonomia e qualidade de vida, devem ter processo de elaboração participativo e valorizar as relações, interações e retroações dos participantes permeado pelo sinergismo do conhecimento intermulti-trans-meta-disciplinar [7,8], proporcionando cuidado à saúde do ser humano de forma mais contextualizada e humanizada [9], e construindo um produto educativo - vídeo, folder, carta, cartilha, outdoor - capaz de promover a aprendizagem significativa.

Desta forma, a gerontotecnologia educativa é considerada instrumento disponível para facilitar o processo de ensino-aprendizagem no âmbito da saúde, proporcionando o desenvolvimento de habilidades e conhecimentos necessários para o cuidado, por aglutinar as experiências cotidianas aos conhecimentos científicos, a fim de provocar intervenções sobre uma situação prática [10]. Dada suas características pedagógicas próprias e atuais, o uso do termo gerontotecnologia faz-se necessário para identificação da abordagem e do público da atividade ou ação educativa de forma rápida e específica.

No Brasil estudos para a construção de gerontotecnologia foram realizados versando sobre cuidados com ostomia [3], doença de Alzheimer [10], condições crônicas [11], dependência física [12], e no campo das engenharias [13] na criação de espaços acolhedores aos idosos, as tecnologias destinaram-se, usualmente, aos idosos e sua família. Contudo, seu uso ainda é incipiente, necessitando de mais estudos que aborde sua construção e potencial educativo.

Cabe destacar que gerontotecnologia, no campo da educação é compreendido como qualquer instrumento novo e educativo que contribua para o cuidado à saúde do idoso. E que foi elaborado considerando as características do processo de envelhecimento e do processo saúde/doença dos idosos envolvidos a fim de que o instrumento seja capaz de facilitar e/ou contribuir para o cuidado ou autocuidado, corresponsabilidade e coparticipação do idoso e sua família [12].

Dado o potencial educativo da gerontotecnologia, se faz necessário expandir o conceito e aplicabilidade da mesma de modo que as inovações e o uso criativo dos instrumentos disponíveis para estimular o pensamento crítico sejam frequentes nas práticas educativas em saúde. Esta tecnologia educativa corrobora as necessidades de atendimento personalizado ao idoso, pois o envelhecimento populacional traz novas perspectivas de gestão e de cuidado que devem ser abarcadas pelos profissionais de saúde.

Diante da gerontotecnologia como nova estratégia educativa e de cuidado que impacta positivamente na educação em saúde aos idosos e com a presença frequente dos mesmos nas atividades desenvolvidas na atenção primária à saúde, encontra-se campo fértil e aberto à construção de gerontotecnologias delineadas nos anseios dos idosos.

Para tanto, objetivamos descrever o processo contextualizado e participativo da construção de uma gerontotecnologia educativa na atenção básica sobre acidente vascular cerebral.

Material e métodos

Tratou-se de uma pesquisa de desenvolvimento metodológico, com abordagem qualitativa. As pesquisas desse tipo almejam desenvolver, adequar, avaliar ou aprimorar um instrumento metodológico, por meio da obtenção, organização e análise de dados [14]. Podem ser utilizadas para avaliar a solidez, confiabilidade e adequabilidade de técnicas de pesquisa ou recursos educativos $[15,16]$.

Desta forma, é o tipo de pesquisa adequada para a construção e adequação de gerontotecnologias $[14,16]$. Cumpre destacar que o levantamento da demanda educativa foi realizado em estudo anterior, em um projeto de iniciação científica, que elencou as temáticas de preferência de 40 idosos de uma Unidade Básica de Saúde escolhida de forma intencional por possuir vínculo com a Instituição de Ensino, localizada no Noroeste do Estado do Paraná. Os idosos foram abordados na sala de espera da unidade enquanto aguardavam atendimento por demanda espontânea ou oferta programada.

Estes 40 idosos foram escolhidos aleatoriamente enquanto aguardavam atendimento na unidade, destes 24 são do sexo feminino (60\%) e 16 do sexo masculino (40\%), a média de idade dos participantes foi de 72 anos, o nível de escolaridade predominante foi ensino fundamental incompleto, totalizando $15(37,5 \%)$ idosos. A maioria $(\mathrm{N}=25-62,5 \%)$ dos idosos eram casados, e consequentemente moravam com seus companheiros, usualmente com mais um familiar seja ele neto (a) ou filho (a). 
Os dados foram coletados durante os meses de janeiro a março de 2016, por meio de entrevista semiestruturada, utilizando-se como instrumento um roteiro elaborado pelas pesquisadoras, dividido em duas partes: a primeira para coletar dados referentes à caracterização sociodemográfica e a segunda com questões abertas referentes às demandas educativas dos idosos. Os depoimentos foram gravados e transcritos na íntegra, e analisados.

As demandas educativas apontadas foram: etilismo, Diabetes mellitus, chikungunya, hipertensão arterial e acidente vascular cerebral (AVC), sendo esta última a escolhida como temática para a elaboração da gerontotecnologia por apresentar maior frequência e se tratar de uma doença relevante à fase do envelhecimento, por sua alta incidência e associação com outras morbidades.

A forma e o local de apresentação da gerontotecnologia foram definidos pelas pesquisadoras com auxílio dos profissionais da unidade de saúde considerando a organização e estrutura da mesma. Desta forma, a apresentação audiovisual na sala de espera foi escolhida pela facilidade e amplitude de ação, já que a referida unidade possui aparelho televisor fixo no ambiente e este espaço possui alto fluxo de pessoas, especialmente idosas.

O presente estudo metodológico, portanto, pautou-se nesta demanda educativa levantada previamente. Participaram da pesquisa como avaliadores da gerontotecnologia elaborada, três profissionais com experiência na área de gerontologia. Para formação desse grupo, foi solicitada a indicação de profissionais capacitados à orientadora do projeto e professora do departamento de enfermagem da Universidade Estadual de Maringá. Como rigor para adequação, foram convidados profissionais com experiência na construção ou avaliação de materiais educativos.

Para tanto, elaboramos este instrumento ancorado nos pressupostos de Manzini [17], dividindo-o em duas partes para sua análise: 1) Análise da forma, sequência e abrangência dos slides; 2) Análise da ação verbal dos textos utilizados nos slides.

Após a adequação pelos profissionais juízes, os idosos presentes na sala de espera da UBS em que a demanda educativa foi levantada, participaram de uma roda de conversa mediada pela gerontotecnologia elaborada, permitindo que também participassem da adequação da forma e conteúdo da mesma.

O estudo em tela, por fazer parte de um estudo maior, possui aprovação do Comitê Permanente de Ética em Pesquisa Envolvendo Seres Humanos da Universidade Estadual de Maringá (parecer no 875.081, CAE 37457414.6.0000.0104), e seguiu todos os preceitos éticos vigentes na Resolução 466/2012 do Conselho Nacional de Saúde.

Os resultados estão organizados seguindo 0 processo de construção da gerontotecnologia, o qual foi permeado pelo diálogo e pela participação dos envolvidos a fim de que o material possuísse maior proximidade com a realidade e atendesse seu potencial educativo.

\section{A elaboração da gerontotecnologia educativa}

Para a construção da tecnologia educativa o idoso foi considerado participante ativo do processo de aprendizado. Portanto, a construção da gerontotecnologia foi conduzida de acordo com as demandas elencadas previamente, buscando a transversalidade de saberes e práticas e a promoção da saúde.

Assim, para a construção de cada etapa da gerontotecnologia educativa e seu processo de adequação, utilizou-se abordagem dialógica por valorizar o conhecimento e demandas apresentadas pelos participantes.

Após a escolha da temática, AVC, a gerontotecnologia educativa foi construída utilizando como recurso didático a apresentação audiovisual elaborada no programa Power Point, versão 2010, do pacote Microsoft@, o qual permite elaborar apresentações com imagens, textos e áudios formando um vídeo.

Assim, o conteúdo educativo foi organizado em 18 slides, formato escolhido por ser de fácil manuseio e por permitir inserções dinâmicas, com a organização de imagens e textos, a fim de apresentar de forma didática, acessível e clara o conteúdo abordado. Ainda, utilizou-se o recurso de gravação de áudio utilizando a fala da própria autora e finalizou-se formando um vídeo. 
Para a organização dos conteúdos as pesquisadoras fizeram leitura sobre a fisiopatologia do AVC em literatura atual e pertinente, organizando os dados em: significado do termo AVC; função dos vasos sanguíneos; permeabilidade dos vasos sanguíneos; tipos de AVC; fatores de risco para o AVC e suas contribuições para o desenvolvimento da doença; sinais e sintomas do AVC; e prevenção do AVC e dos agravos.

Dessa forma, a tecnologia foi elaborada respeitando a sequência dos fatores desencadeantes do AVC em nosso organismo. Tal organização favoreceu melhor entendimento dos participantes e ampliação das discussões no local do estudo.

Para a escrita dos slides foi utilizada linguagem simples e acessível ao público, por meio do uso de frases curtas e pela alternância entre a palavra escrita e imagens, a fim de facilitar a compreensão das informações e do material. Utilizaram-se comparações para facilitar o entendimento do conteúdo, como os vasos sanguíneos comparados às rodovias, buscando a associação de conhecimentos teóricos e práticos que possam contribuir efetivamente para o aprendizado e a qualidade de vida.

\section{A adequação por juízes}

O material na versão de vídeo foi avaliado para adequação por experts na área. Para tal, foram escolhidos três juízes com experiência na temática e no desenvolvimento de tecnologias educativas, estes são enfermeiros com doutorado na área da saúde e atuantes na docência. Para tal enviou-se por e-mail um convite formal para participação no estudo e após o aceite foram enviadas as instruções sobre o trabalho e o processo de adequação, 0 instrumento de adequação e a gerontotecnologia elaborada.

Os juízes avaliadores enviaram as sugestões de alterações no texto: grafia, gramática, tipo e localização das imagens, cinco dias após o envio da gerontotecnologia, a fim de melhorar a aplicabilidade do conteúdo ao público alvo, buscando atingir o objetivo inicial.

As sugestões dos experts se pautaram em: reorganização dos ícones de áudio e das figuras; definir como padrão cor do fundo preta e da fonte branca; padronizar o tipo e tamanho de fonte em todos os slides; e substituir alguns textos para linguagem de fácil compreensão para leigos.

O instrumento de adequação foi elaborado seguindo as premissas de Mazini [17] e continha espaço para identificação profissional e um quadro para avaliação de cada slide com escala do tipo Likert (insatisfatório, parcialmente satisfatório, satisfatório e altamente satisfatório), solicitando-se justificar quando assinalado insatisfatório ou parcialmente satisfatório. Quando pelo menos um dos juízes apontou parcialmente satisfatório ou insatisfatório foi considerada discordância entre os juízes [11].

Dessa forma, doze slides não necessitaram de alteração e seis slides foram reescritos, seguindo as sugestões feitas pelos juízes especialistas, a fim de garantir o aperfeiçoamento da gerontotecnologia.

\section{Roda de conversa e adequação pelos idosos participantes}

Após a adequação da gerontotecnologia educativa pelos profissionais, foi realizada uma roda de conversa com os idosos que aguardavam atendimento na sala de espera da unidade de saúde. A gerontotecnologia educativa foi apresentada e iniciada discussão reflexiva sobre o tema e a forma da tecnologia, realizando o levantamento de sugestões e adequações na perspectiva dos idosos.

Em seguida, foi realizada adequação de aparência e conteúdo seguindo os apontamentos dos idosos. Por fim, os idosos aprovaram a gerontotecnologia reconhecendo-a como instrumento terapêutico capaz de veicular conhecimento e aprendizagem ampliando sua compreensão sobre o processo saúde-doença.

Durante a roda de conversa, os participantes referiram que os slides foram capazes de atingir o objetivo proposto, pois as palavras utilizadas foram adequadas e objetivas, os slides estavam organizados de modo dinâmico e o assunto foi considerado de extrema importância para o cotidiano. Relataram também, que a forma como foi aplicada a gerontotecnologia vídeo na televisão da sala de espera - deveria ser mais utilizada, pois é rápida e fácil facilitando o processo de ensino-aprendizado de temas relativos à saúde.

Referiram também, que o alerta para os riscos que as doenças podem causar e como elas agem no organismo foram de grande valia, pois desconheciam esse tipo de informação e não sabiam quais eram os sintomas e como identificar uma pessoa que está sofrendo um AVC 
próximo a eles, e que a gerontotecnologia contribuiu para a prevenção e identificação precoce do AVC.

As discussões que ocorreram na roda de conversa evidenciaram que os participantes possuem entendimento de que os comportamentos de um indivíduo geram reflexos para a sua saúde. Assim, as pessoas são responsáveis pelo seu autocuidado a fim de evitar doenças ou amenizar as consequências em seu corpo. Portanto, o desenvolvimento do cuidado gerontológico deve pautar-se na compreensão do idoso enquanto cidadão e protagonista do seu cuidado e/ou tratamento. E para ser emancipador e verdadeiramente promotor da saúde deve-se romper com a lógica culpabilista hegemônica durante os processos educativos, como almejou este estudo.

Discussão

A elaboração participativa de uma gerontotecnologia auxiliou no processo de ensinoaprendizagem dado seu potencial educativo, pois se pautou nas demandas reais dos idosos. Quando se utiliza de ferramentas tecnológicas de informação e comunicação têm-se potencial para ampliar as práticas colaborativas e aprendizagem autônoma [15]. Entre as diversas ferramentas, o uso de vídeos educativos ganha destaque com o público idoso, especialmente quando construído especificamente para eles e sua vantagem foi reafirmada no estudo em tela $[18,19]$.

O rigor metodológico e científico da construção da gerontotecnologia garante seu potencial educativo e a difere de materiais educativos convencionais, pois é feita - forma e conteúdo - direcionada para o público específico [8,12], com respaldo pedagógico e da saúde, no caso.

Neste contexto, o uso do Microsoft Power Point $\AA$ para a construção dos slides e do vídeo possibilitou desenvolver uma tecnologia adequada e de fácil compreensão. Por tratar-se de uma ferramenta de baixo custo e de fácil manuseio, permitiu construir uma tecnologia educativa de qualidade, atestada por juízes experts e representantes do público-alvo. Este processo foi fundamental para garantir a eficácia educativa do material [18,20].

Acrescenta-se que o uso da tecnologia audiovisual possivelmente repercutirá de modo positivo no conhecimento e no autocuidado dos idosos e das famílias, pois permitiu ilustrar e complementar os conhecimentos já existentes [17]. De fato, argumenta-se que os recursos audiovisuais são potentes instrumentos didáticos e tecnológicos que aglutinam, em um único objeto de promoção do conhecimento, vários elementos, tais como: imagens, texto e som, estimulando o conhecimento, a autonomia e a consciência crítica [18-20], tal qual apreendemos nesse percurso.

Desta forma, a gerontotecnologia desenvolvida de forma dialógica e participativa apresenta-se como estratégia capaz de estimular o autocuidado e o cuidado de si pelo empoderamento do idoso, emergindo como inovação para a promoção da saúde e a emancipação dos envolvidos [21-24].

Quanto ao recurso didático, o vídeo elaborado pelas pesquisadoras é uma estratégia que facilita e potencializa o processo de ensino-aprendizado por apresentar de forma clara e dinâmica informações complexas e de atrelar saberes científicos e fisiopatológicos com saberes populares e vivenciados, permeado pela discussão da temática na perspectiva dos envolvidos, garantindo a proximidade do tema e da linguagem com o universo pesquisado $[20,21]$.

A adoção da estratégia pedagógica baseada em dinâmicas de grupo para eleger a demanda educativa e para validar a gerontotecnologia elaborada propiciou o encontro do saber técnico-científico com a realidade vivenciada rompendo-se com o modelo tradicional e vigente no campo da saúde, o da transmissão de informações. Desse modo, inseriu-se a abordagem dialógica no contexto da saúde, esta considera que tanto o educador quanto o educando possuem conhecimentos e experiências que devem ser considerados durante a prática educativa [22] e enriquecem e dão sentido ao processo de ensino-aprendizagem e ao cuidado.

Pautando-se na dialogicidade e na imersão na realidade, a sala de espera da unidade foi o local de estudo escolhido para o levantamento dos temas e adequação da gerontotecnologia pelos idosos, por se tratar de um espaço dinâmico de encontro daqueles que buscam ou aguardam qualquer tipo de atendimento na atenção básica, e que possibilita 0 encontro e o diálogo dos usuários e dos profissionais, além da comunhão da sociedade com o serviço [23]. 
A abordagem em grupo foi a estratégia escolhida para estimular o diálogo e a comunhão dos idosos e profissionais, pois se delineia nas necessidades e saberes dos envolvidos sendo a principal estratégia educativa utilizada para a promoção da saúde preconizada pelas políticas de saúde que se referem a educação popular, especialmente para a população idosa $[9,13,21]$.

Além disto, as atividades em grupos possibilitam ampliar o cuidado, por vezes focado na atenção individual e centradas no controle das doenças crônicas, e potencializar uma abordagem mais abrangente de saúde por meio do diálogo, do vínculo e da construção coletiva, fortalecendo a rede de apoio social e mobilizando as pessoas na busca de autonomia e autocuidado [21].

\section{Conclusão}

O percurso metodológico de construção e adequação da gerontotecnologia educativa na temática acidente vascular cerebral mostrou-se oportuna e possível para abordagem de idosos em sala de espera de Unidades Básicas de Saúde.

O caráter dialógico foi preservado, bem como a participação efetiva do público-alvo, fazendo-o ativo no processo de educação em saúde.

Durante o encontro, notou-se que os participantes não possuíam conhecimento sobre a fisiopatologia da doença apresentada, nem a associação dos fatores de risco com a doença. A gerontotecnologia possibilitou inserir e reforçar os conhecimentos pré-existentes e também estimulou reflexão sobre as repercussões do AVC.

O estudo demonstra que a construção de gerontotecnologias educativas no contexto de trabalho e voltado às necessidades da população é possível, pode ser feito com recursos relativamente simples e acessíveis, e deve ser estimulado entre os profissionais de saúde, pelo seu potencial transformador.

Os aspectos limitantes do estudo foram o pequeno número de idosos que participaram da roda de conversa para adequação da gerontotecnologia, em virtude de faltas frequentes nas consultas previamente agendadas na unidade de saúde, e a escolha da temática por tratar-se de uma demanda local.

Cabe destacar a importância de se adotar e difundir o uso do termo gerontotecnologia para facilitar a localização de estudos que buscam desenvolver tecnologias específicas aos idosos, com características de manter a autonomia e qualidade de vida dos mesmos.

\section{Agradecimentos}

À Secretaria Municipal de Saúde de Maringá e aos integrantes do "Grupo de Estudos e Pesquisas em Práticas Educativas em Saúde" pelo apoio, parceria e colaboração. E ao Conselho Nacional de Desenvolvimento Científico e Tecnológico (CNPq) pela bolsa de iniciação cientifica para a realização desse estudo.

\section{Referências}

1. IBGE. Instituo Brasileiro de Geografia e Estatística. Coordenação de População e Indicadores Sociais. Síntese de indicadores sociais: uma análise das condições de vida da população brasileira. Rio de Janeiro: IBGE; 2015.

2. Crichi KM, Polaro SHI, Gonçalves LHT. Ações de Enfermagem e determinantes do envelhecimento ativo. Enfermagem Brasil 2016;15(6):307-15.

3. Borba AKOT, Marques APO, Leal MCC, Ramos RSPS. Práticas educativas em diabetes Mellitus: revisão integrativa da literatura. Rev Gaúch Enferm 2012;33(1):16976.

4. Brasil. Ministério da Saúde. Secretaria de Vigilância em Saúde. Doenças cardiovasculares causam quase 30\% das mortes no País. [citado 2017 Jul 2017]. Disponível em: http://www.brasil.gov.br/saude/2011/09/doencas-cardiovascularescausam-quase-30-das-mortes-no-pais.

5. Castro DC, Nunes DP, Pagotto V, Pereira LV, Bachion MM, Nakatani AYK. Incapacidade funcional para atividades básicas de vida diária de Idosos: estudo populacional. Ciênc Cuid Saúde 2016;15(1):109-17. 
6. Áfio ACE, Balbino AC, Alves MDS, Carvalho LV, Santos MCL, Oliveira NR. Análise do conceito de tecnologia educacional em enfermagem aplicada ao paciente. Rev Rene 2014;15(1):158-65.

7. Araújo SNM, Santiago RF, Barbosa CNS, Figueiredo MLF, Andrade EMLR, Nery IS. Tecnologías orientadas al cuidado del anciano en los servicios de salud: una revisión integradora. Enferm Global 2017;16(2):579-95.

8. Ilha S, Santos SSC, Backes DS, Barros EJL, Pelzer MT, Oliveira AMN. Tecnologia cuidativo-educacional na doença de Alzheimer e no apoio ao idoso/família: perspectiva dos docentes e discentes. Esc Anna Nery 2017;21(2):e20170039.

9. Mendonça ERT, Aires LFA, Amaro MO, Moreira TR, Henriques BD, Almeida LC. A experiência de oficinas educativas com idosos: (re)pensamento práticas à luz do pensamento Freireano. Rev APS 2013;4(4):479-84.

10. Barros EJL, Santos SSC, Gomes GC, Erdmann AL. Gerontotecnologia educativa voltada ao idoso estomizado à luz da complexidade. Rev Gaúch Enferm 2012;33(2):95101.

11. Polit $D$, Beck $C$. Fundamentos de pesquisa em enfermagem: avaliação de evidências para a prática da enfermagem. $7^{\underline{a}}$ ed. Porto Alegre: Artmed; 2011.

12. Santos SSC, Gautério DP, Vidal DAS, Rosa BM, Zortea B, Urquia BS. (In)dependência na realização de atividades básicas de vida diária em pessoas idosas domiciliada Rev Rene 2013; 14(3):579-87.

13. Machado MMB. Estudo sobre a adequação hoteleira para atender o segmento da terceira idade [monografia]. 2008. 124 f. Brasília: Universidade de Brasília; 2008.

14. Martins MC, Veras JEGLF, Uchoa JL, Pinheiro NC, Vieira NFC, Ximenes LB. Segurança alimentar e uso de alimentos regionais; validação de um álbum seriado. Rev Esc Enferm USP 2012;46(6):1054-1361.

15. Lima DVM. Desenhos de pesquisa: uma contribuição ao autor. OBJN 2011;10(2):1-18.

16. Manzini EJ. Considerações sobre a elaboração de roteiros para entrevista semiestruturada. In: Marquezine MC, Almeida MA, Omote S. Colóquios sobre pesquisa em educação especial. Londrina: Eduel; 2003. p.11-25.

17. Dalmolin A, Girardon-Perlini NMO, Coppetti LC, Rossato GC, Gomes JS, Silva MEN. Vídeo educativo como recurso para educação em saúde a pessoas com colostomia e familiares. Rev Gaúch Enferm 2016;37(esp):e68373.

18. Tam JW, Son CV, Dyck D, Schmitter-Edgecombe M. An educational video program to increase aging services technology awareness among older adults. Patient Educ Couns 2017;100(8):1564-71.

19. Costa SRS, Duqueviz BC, Pedroza RLS. Tecnologias digitais como instrumento mediadores da aprendizagem dos nativos digitais. Psicol Esc Educ 2015;19(3):603-10.

20. Schulz R, Wahl H-W, Matthews JT, Dabbs AV, Beach SR, Czaja SJ. Advancing the aging and technology agenda in gerontoly. Gerontologist 2015;55(5):724-34.

21. Sousa LM, Assis M. Educação popular em saúde e grupos de idosos: revisão sobre princípios teórico-metodológicos das ações educativas em promoção da saúde. Rev APS 2012;15(4):443-53.

22. Amaral MA, Amorin MMA, Torres HC, Abreu CRT. Oficinas educativas na atenção primária de saúde para promoção do autocuidado em diabetes mellitus. Rev APS 2014;17(1):58-64.

23. Emmi DT, Pires MJM. Acolhimento e educação em saúde na sala de espera: avaliação da contribuição das ações para o atendimento odontopediátrico. Rev Aten Saúde 2016;14(48):62-7. 